\title{
Preface
}

\section{Fluid Resuscitation in Burn \\ Patients: Current Care and New \\ Frontiers}

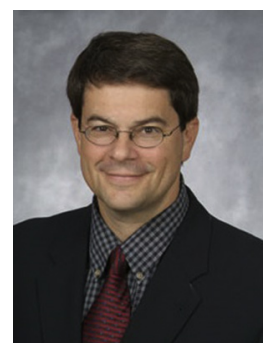

Kevin N. Foster MD, MBA, FACS

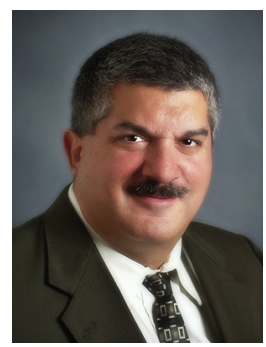

Daniel M. Caruso, MD, FACS

Editors

The recognition that burn injury causes a profound inflammatory response accompanied by movement of intravascular fluid into the extravascular space and that this intravascular fluid loss must be aggressively replaced by intravenous fluid administration was one of the key advances in burn care in the last century. Subsequent studies of fluid resuscitation following burn injury demonstrated improved patient outcomes. Since that time, fluid resuscitation in burn patients has been a fertile field for both basic science and clinical research.

Current clinical care for fluid resuscitation of burned patients consists of determining the extent of the burn injury, determining the volume or rate of fluid administration based on one of several validated fluid formulas, and administering fluid as a balanced salt solution with or without colloid. There is no recognized specific standard of care for burn resuscitation. Subsequent fluid administration is based on patient response, primarily urine output. Additional modifications to the fluid resuscitation strategy may be made based on individual patient characteristics such as delayed presentation and resuscitation, the presence of inhalation injury, the presence of comorbidities such as cardiac and/or pulmonary disease, and the development of conditions such as acute kidney injury.

This issue of Critical Care Clinics reviews the current status of fluid resuscitation in burn patients, including existing knowledge of the pathophysiologic mechanisms of burn shock, the use of colloid in resuscitation paradigms, monitoring the success of fluid resuscitation, and fluid resuscitation in pediatric patients. Additional topics to be covered include the utility of vitamin $C$ in acute resuscitation, caring for patients with complicated resuscitations, treatment options when standard clinical fluid resuscitation paradigms fail, and the recent issue of so-called fluid creep and overaggressive resuscitation. Finally, this issue also presents the relatively novel concept of 
xvi Preface

protocolized and/or computerized models of resuscitation and explores novel and unique future therapies in burn resuscitation.

Kevin N. Foster, MD, MBA, FACS The Arizona Burn Center University of Arizona College of Medicine 2601 East Roosevelt Phoenix, AZ 85008, USA

Daniel M. Caruso, MD, FACS Department of Surgery University of Arizona College of Medicine The Arizona Burn Center Maricopa Medical Center 2601 East Roosevelt Phoenix, AZ 85008, USA

E-mail addresses:

kevin_foster@dmgaz.org (K.N. Foster) danile_caruso@dmgaz.org (D.M. Caruso) 